\section{A New Unifying Biparametric Nomenclature that Spans All of Chemistry}

by Seymour B. Elk

Elsevier, 2004, ISBN 0-444-51685-9

\section{reviewed by Kevin Thurlow}

I read the title of this book with some trepidation-what could it mean? The subtitle is "The science of incorporating over 2000 new names to a base of over 42 million compounds while still maintaining order." The author, Seymour B. Elk, is concerned that there are different forms of nomenclature for "inorganic chemistry," "organic chemistry," and "polymer chemistry," etc. Elk makes the entirely reasonable point that as new chemicals are discovered, the boundaries between these different areas become somewhat blurred, and there are communication difficulties among chemists.

Therefore, Elk proposes an unified scheme, where the name is based entirely on the geometric structure. The author has a background in mathematics and computers, as well as chemistry, and his proposed system reflects this. He points out obvious faults in IUPAC nomenclature (e.g., "fluorine" and "fluorene" sound the same, but are entirely different; and "polyethylene" is used instead of "polymethylene"). He also draws attention to IUPAC's use of alphabetical order to set priorities. He says that using size would give I > $\mathrm{Br}>\mathrm{Cl}>\mathrm{F}$, or using reactivity would give $\mathrm{F}>\mathrm{Cl}>\mathrm{Br}$ $>$ I. This works fine for simple examples of course.

The claimed advantages of his system include the following: one set of rules that can easily be expanded if new forms of structure are discovered; recognition that chemistry is three-dimensional; removal of traditional prefixes and suffixes; creation of "single, unified, systemic formulations for addending modules at specified locations to an evolving skeletal base" (which avoids consulting long tables of parent names); elimination of oxidation number; new ways of dealing with tautomers and aromatic compounds; and improved systems for boranes and polymers. In brief, the idea is to have one system to cover all chemical names. This is a laudable ideal, but is it possible or even desirable in everyday circumstances?

The system espoused is a line system, like Wiswesser and SMILES, and also under development in the InChl project. As such, it really relies on computers for creation and understanding of "names." (The author does not like to "name" chemicals, he "nomenclates" them!) He is certainly not fond of IUPAC, but his knowledge is a little hazy as he says that 'butan-1-ol' is a British name and '1-butanol' is IUPAC. He reasonably says that multiple primes and Greek letters are a nuisance, but then uses $\alpha$ and $\beta$ and $N$ (Hebrew "aleph") himself. He gives (page 32) the "IUPAC" name 3(2-propynyl),5-methenyl-oct-1,2-diene,6-yne and its equivalent in his system, which is:

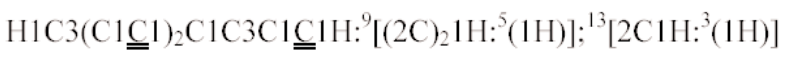

The ' $\underline{\underline{C}}^{\prime}$ requires some juggling of the keyboard, and means a non-terminal ' $\mathrm{CH}_{2}$ ' group. (' $\mathrm{C}$ ' would be a nonterminal $\mathrm{CH}$ group.) $\mathrm{He}$ carries the rule for selecting longest chain to extremes, including the terminal hydrogen atoms. The example above shows a drawback to his system. The "IUPAC" name has errors, but you can still work out what it means. Any error in the second "name" would be difficult to detect and correct.

I doubt that any one system of nomenclature can meet universal approval. Sometimes, you want structural diagrams, or CAS numbers, or IUPAC names, or INNs for drugs, or ISO pesticide names, or INCI cosmetic names, or hazard warning labels, or abbreviations... The list is endless. You use whatever is best for the given audience and the given situation. Any nomenclature system has failings. In the system proposed in this book, for example, you cannot easily speak the name, and a small typing error would cause complete confusion.

Unfortunately, this book has a large number of typographical errors. "Flouro" instead of "fluoro" repeatedly. The layout is also confusing. It is not always clear which "names" refer to which diagrams, and there are frequent, lengthy footnotes, up to half a page long. The author has a tendency to circumlocution, which may irritate some readers.

To summarize, it is an interesting system that Elk proposes, which has good and bad points, like all systems of nomenclature. It appears to succeed in producing a naming system for everything, but the "names" produced are incredibly complicated, and not, in my view, beneficial for communication.

Kevin Thurlow <kjt@lgc.co.uk> from LGC, Ltd. Chemical Nomenclature Advisory Service in Teddington, Middlesex, UK, is also a member of the Advisory Committee of the IUPAC Chemical Nomenclature and Structure Representation Division.

\section{Siiif http://books.elsevier.com/elsevier/?ishn=0444516859}

